\title{
ARTICLE
}

Received 18 Sep 2012 | Accepted 26 Nov 2012 | Published 27 Dec $2012 \quad$ DOl: 10.1038/ncomms2331

\section{From chaos to selective ordering of vortex cores in interacting mesomagnets}

\author{
S. Jain ${ }^{1}$, V. Novosad', F.Y. Fradin', J.E. Pearson', V. Tiberkevich², A.N. Slavin² \& S.D. Bader ${ }^{1,3}$
}

A spin vortex consists of an in-plane curling magnetization and a small core region ( $\sim 10 \mathrm{~nm}$ ) with out-of-plane magnetization. An oscillating field or current induce gyrotropic precession of the spin vortex. Dipole-dipole and exchange coupling between the interacting vortices may lead to excitation of collective modes whose frequencies depend on the core polarities. Here we demonstrate an effective method for controlling the relative core polarities in a model system of overlapping $\mathrm{Ni}_{80} \mathrm{Fe}_{20}$ dots. This is achieved by driving the system to a chaotic regime of continuous core reversals and subsequently relaxing the cores to steady-state motion. It is shown that any particular core polarity combination (and therefore the spectral response of the entire system) can be deterministically preselected by tuning the excitation frequency or external magnetic field. We anticipate that this work would benefit the future development of magnonic crystals, spin-torque oscillators, magnetic storage and logic elements.

\footnotetext{
${ }^{1}$ Materials Science Division, Argonne National Laboratory, Argonne, Illinois 60439, USA. ${ }^{2}$ Department of Physics, Oakland University, Rochester, Michigan 48309, USA. ${ }^{3}$ Center for Nanoscale Materials, Argonne National Laboratory, Argonne, Illinois 60439, USA. Correspondence and requests for materials should be addressed to V.N. (email: novosad@anl.gov).
} 
$\mathrm{V}$ ortices are observed in the statics and dynamics of a variety of physical systems, such as fluids and plasma ${ }^{1}$, optics $^{2}$, superconductors ${ }^{3}$ and magnetic materials ${ }^{4}$. In patterned mesoscopic ferromagnets, the ground state of the static magnetization can have a form of a vortex that consists of a large region of in-plane curling magnetization (clockwise or anticlockwise sense of rotation or chirality) and a small core region $(\sim 10 \mathrm{~nm})$ with out-of-plane magnetization, which can be pointing either up $(+1)$ or down $(-1)$ (positive or negative core polarity $p$ ). When the vortex core is perturbed from its equilibrium state by an in-plane oscillating field or current, it begins a rotational (gyrotropic) oscillation with a characteristic eigenfrequency in the subgigahertz range $\mathrm{e}^{5-7}$. In a magnetic dot that has a single vortex-state, the eigenfrequency of the gyrotropic oscillations of the vortex core for both directions of the core polarity $(p= \pm 1)$ is the same. By contrast, in an array of coupled elements, the dynamic dipolar interaction eliminates this frequency degeneracy and results in collective gyrotropic excitations with different eigenfrequencies that depend on both the relative polarities and chiralities of the individual vortices ${ }^{8-11}$. An array of interacting magnetic dots form a vortex magnonic crystal $^{12,13}$, similar to the case of coupled, uniformly magnetized elements ${ }^{14,15}$. The system can have a dynamically reconfigurable ground state characterized by different relative polarities and chiralities of the individual dots; and it can have a corresponding dynamically controlled spectrum of collective excitation modes that determine the microwave absorption of the crystal. The development of effective methods for dynamic control of the ground state in vortex-type magnonic crystals is fundamentally interesting and could also be important for possible microwave applications. Vortex chirality has been controlled in confined geometries using various techniques ${ }^{16,17}$. Manipulation of the vortex core polarity has been shown in individual elements by resonant in-plane ${ }^{18,19}$ and perpendicular magnetic fields ${ }^{20}$, rotating magnetic fields ${ }^{21}$, pulsed currents ${ }^{22}$ and spin-polarized currents $^{23}$. However, control of the vortex core polarities in interacting vortex-state magnetic dots remains a challenge.

In this work, we demonstrate an effective method for controlling the relative vortex core polarities in a model system of connected (slightly overlapping) vortex-state permalloy $\left(\mathrm{Ni}_{80} \mathrm{Fe}_{20}\right)$ magnetic dots. This method also permits the effective selection of a specific dynamic mode of microwave absorption. We utilize the geometry of connected double-dot structures because they have the potential to form building blocks of vortex magnonic crystals ${ }^{12,13}$. An advantage of the double-dot overlap structure is the strong dynamic interaction between the vortex cores and, consequently, the large difference between the eigenfrequencies of the gyrotropic dynamic modes that correspond to the same and opposite orientations of the interacting cores. In the remanent state of this structure, the configurations with the same $\left(p_{1} p_{2}=+1\right)$ or opposite $\left(p_{1} p_{2}=-1\right)$ core polarities are equally probable. Therefore, the selection of a particular dynamic mode in this system is achieved by first driving the system into a chaotic regime of continuous vortex core reversals via a large-amplitude external driving signal that has the same frequency as one of the dynamic gyrotropic modes of the system. Then, the driving amplitude is reduced so that the system relaxes to the state corresponding to the other collective gyrotropic mode that has a different eigenfrequency; therefore, the system can no longer resonantly absorb energy from the driving signal.

\section{Results}

Sample structure and measurement setup. Permalloy $\left(\mathrm{Ni}_{80} \mathrm{Fe}_{20}\right)$ double-dot structures with dimensions of $2 \times 1 \times 0.048 \mu \mathrm{m}^{3}$ and vertical contact length of $740 \mathrm{~nm}$ were fabricated on top of shorted coplanar waveguide (CPW). Representative atomic force microscopy image for a dot pair is shown in Fig. 1a. The signal line of the CPW consists of $>1,000$ of such dot-pairs. Microwave measurements were performed in reflection mode using a vector network analyser as the current source. Figure 1b shows the schematic of the CPW, where the radio frequency (RF) field is applied along the long axis of the system ( $x$ axis). The absorption spectrum for the as-grown sample at remanence for $-15 \mathrm{dBm}$ of input microwave power $\left(P_{\text {Low }}\right)$ is shown in Fig. 1c. This system of two vortex cores has an equal probability of having either of the two polarization combinations ${ }^{24}$. The two absorption peaks in Fig. $1 c$ correspond to opposite $\left(p_{1} p_{2}=-1\right)$ and same $\left(p_{1} p_{2}=+1\right)$ polarity combinations. It should be noted that the measured spectra is the response from $>1,000$ dot pairs on the waveguide. As the structures are fabricated using electronbeam lithography technique, we believe that the effect of size variation on the resonant frequencies of the coupled modes is within the line-width of each excited mode. The eigenfrequencies for the two polarity combinations are $265 \mathrm{MHz}\left(\omega_{\uparrow \downarrow}\right)$ and $305 \mathrm{MHz}\left(\omega_{\uparrow \uparrow}\right)$, respectively. This spectrum is similar to the response from an array of elliptical particles ${ }^{24}$. The structure of the collective gyrotropic modes follows from the symmetry of the double-dot system (Supplementary Fig. S2). For the $p_{1} p_{2}=-1$ state, the two vortex cores gyrate out-of-phase with each other along the direction of RF excitation ( $x$-axis) and in-phase in the orthogonal direction ( $y$-axis). However, for the $p_{1} p_{2}=+1$ state, the two cores gyrate in-phase for both directions, as schematically illustrated in Fig. 1c. Because the chiralities of the two vortices in a double-dot system are always opposite to each other, the $p_{1} p_{2}=-1$ mode is linearly polarized (the net magnetic moment oscillates along $x$-axis), whereas the polarization of the $p_{1} p_{2}=+1$ mode is elliptical (the net oscillating magnetic moment has both $x$ and $y$ components).

Demonstration of polarity control. To explore the phenomenon of vortex core reversal in coupled vortices, the double-dot structures were driven at a high microwave power of $10 \mathrm{dBm}$ $\left(P_{\mathrm{High}}\right)$ at the resonance frequency of one of the collective modes. When the power was subsequently reduced to a minimum accessible value of $P_{\text {Low }}$, which was used to probe vortex excitations in the linear regime, only one absorption peak that corresponded to the second collective mode was observed in the frequency spectrum. This result indicates that when the system is excited by a large-amplitude signal at the frequency of $\omega_{\uparrow} \downarrow$, with the reduction of the driving amplitude, the system relaxes to the ground state of core polarities that correspond to the collective mode with the frequency of $\omega_{\uparrow \uparrow}$ and vice versa.

The representative absorption spectra for two different excitation frequencies of $\omega_{\uparrow \downarrow}$ (red curve) and $\omega_{\uparrow \uparrow}$ (blue curve) are shown in Fig. 1d. Indeed, only one absorption peak, corresponding to the collective mode that is non-resonant with the frequency of the driving signal, is present in each low-amplitude spectrum of the system. In the following, the excitation process of the vortex core motion with a large-amplitude external signal $P_{\text {High }}$ and the subsequent reduction of the signal amplitude to $P_{\text {Low }}$ at a constant driving frequency corresponding to one of the collective modes will be termed as the initialization procedure. The absorption spectra obtained after this procedure will be measured at $P_{\text {Low }}$ to identify the dynamic collective modes (corresponding to a particular combination of vortex polarities) present in the system.

Micromagnetic modelling. To simulate the experimental results, the gyrotropic motion of the vortex cores in the two dots were 

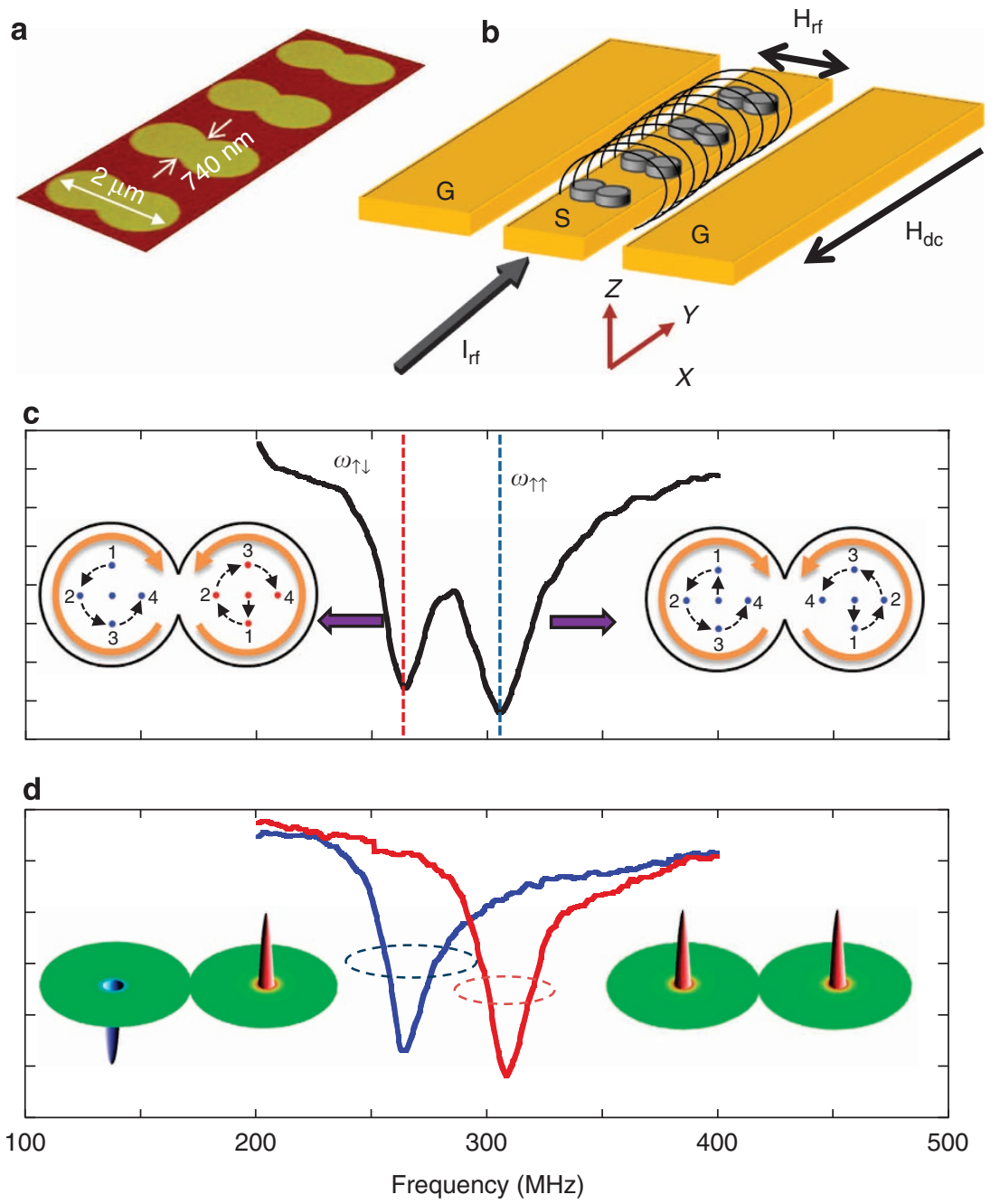

Figure 1 | Absorption spectra before and after mode selection. (a) Atomic force microscopy image of the connected double-disk structure with dimensions of $2 \times 1 \mu \mathrm{m}^{2}$. (b) Schematic of the CPW with connected double disks. (c) Absorption spectrum obtained at zero magnetic field with two absorption peaks corresponding to $p_{1} p_{2}=-1$ and +1 at 265 and $305 \mathrm{MHz}$, respectively. The vortex core gyration in each disk is also illustrated. The solid line illustrates the chirality of the in-plane magnetization. The dashed lines define the direction of vortex core trajectories. (d) Absorption spectra obtained after high RF-amplitude treatment at fixed frequencies of $\omega_{\uparrow \downarrow}$ (red curve) and $\omega_{\uparrow \uparrow}$ (blue curve). The insets show the resultant vortex core polarity combination in the disks.

excited at a high RF amplitude of 52 Oe (sufficiently strong to induce the vortex core reversal), which was then gradually reduced to $2 \mathrm{Oe}$ in steps of 0.1 Oe per cycle. Figure 2 shows the time evolution of the average magnetization when the system was excited at $\omega_{\uparrow \uparrow}$ (Fig. 2a) and $\omega_{\uparrow \downarrow}$ (Fig. 2b). The initial combination of the vortex core polarities can be chosen either parallel or antiparallel. One can easily observe that at sufficiently high RF fields, the two vortex cores undergo continuous reversals, as seen from the chaotic and unsynchronized oscillations of magnetization. However, below a certain RF field, both the $x$ and $y$ components of the dot magnetization stabilize to steady-state oscillations corresponding to a polarity combination of the nonresonant mode. Importantly, this reveals that the resultant state of the system does not depend on the input ground state of vortex core polarities; it only depends on the initialization frequency. Therefore, the final configuration of the system is completely decoupled from the driving signal and cannot absorb energy from this signal.

The transition from chaotic to synchronized vortex core motion, marked as the shadow region in Fig. 2a,b, is of particular interest. This region (shadow area in Fig. $2 \mathrm{~b}$ ) is presented in Fig. 3a. It has been established that the dynamically-driven core reversal is mediated by a series of complex processes: nucleation of a transient vortex-antivortex pair followed by the annihilation of the original vortex with the newly formed antivortex ${ }^{18,23}$. Apart from the critical velocity and critical radius defining the criteria for the core reversal, a well-defined threshold value of the exchange energy is also necessary for this process $^{25}$. Therefore, to quantify the core reversal occurring in the interacting vortices, the exchange energy of the coupled system was considered and the graph of this energy is shown in Fig. 3a. Each peak in the exchange energy is associated with a vortex core reversal. Because the average magnetization response $\left(M_{X}\right.$ and $\left.M_{Y}\right)$ does not directly provide information about the core reversals in each dot, the magnetization components of each dot were separately deduced from the simulations. These components are shown in Fig. 3b,c, respectively. Surprisingly, we observed that in a particular period of time, only one core in the double-disk system undergoes reversal, whereas the other one performs a steadystate rotational motion. This result is surprising because the system is continuously driven using high oscillating fields and 


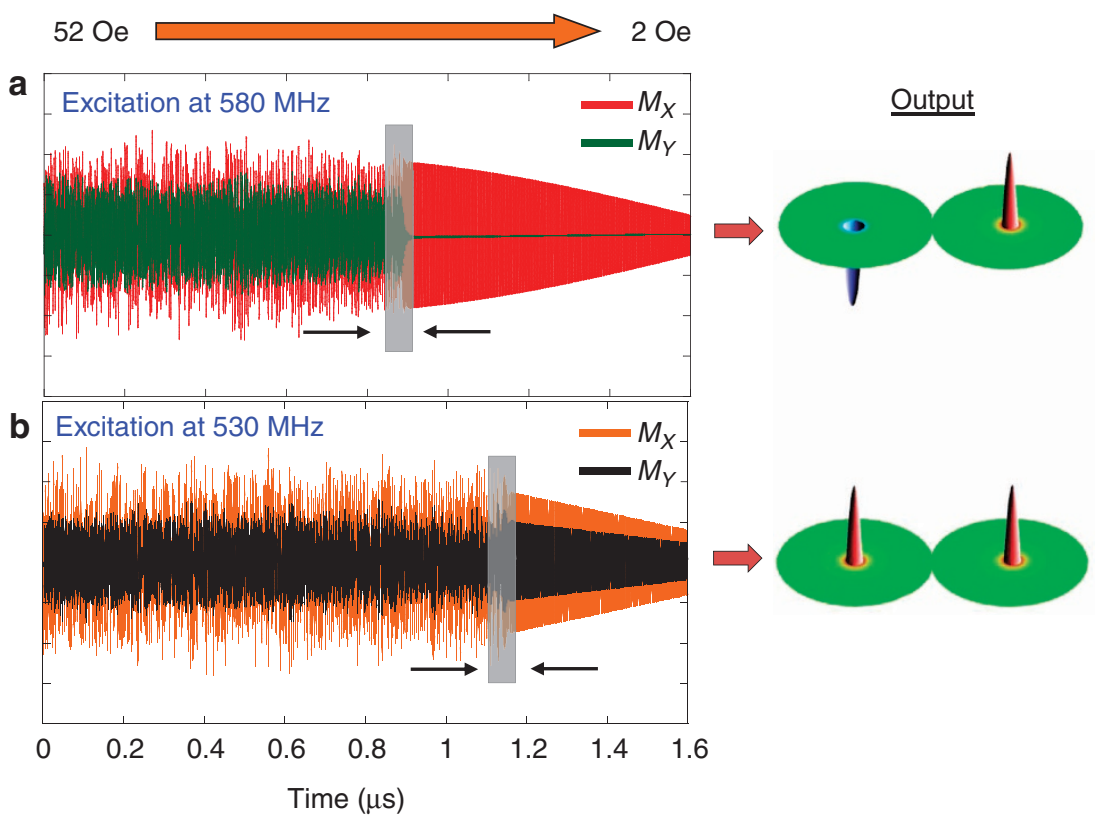

Figure 2 | Demonstration of mode selection using micromagnetic simulations. Micromagnetically simulated time evolution of the magnetizations in the connected double-disk structures at two different excitation frequencies of (a) $580 \mathrm{MHz}$ and (b) $530 \mathrm{MHz}$, while varying the RF-amplitude from 52 to 2 Oe. The final output polarization state of the system is independent of the input combination (which can be either +1 or -1 ) and is only dependent on the excitation frequency.

only one core undergoes several reversal processes. For instance, from $t_{1}=2-38 \mathrm{~ns}$, the core in dot 2 undergoes continuous core reversals, whereas the core in dot 1 rotates with the same frequency as the driving frequency. The vortex core trajectories for this time span are shown in Fig. $3 \mathrm{~d}$. After $\sim 38 \mathrm{~ns}$, there is a transfer of energy between the cores, and the core in dot 1 begins to reverse. The respective core trajectories for this state are shown in Fig. 3e. The dot whose vortex core undergoes reversal is indicated by grey regions in Fig. 3b,c. This behaviour is remarkable because it demonstrates that a systematic reversal pattern exists even in a quasi-chaotic reversal regime (to investigate this phenomenon, an analytical approach was utilized; the detailed description of this approach is provided in Supplementary Discussion). It was observed that the relative phase difference between the two coupled modes determines the particular core that undergoes reversals. The dynamics induced due to this reversal process forces the phase difference to change its sign and consequently, the second core begins to reverse. This transfer process continues until the excitation amplitude is reduced. A particular core continues to reverse for a characteristic time $T$ that is on the order of the inverse of the mode frequency difference, $T \sim 1 / \Delta \omega$.

An energy transfer mechanism was previously observed in separated dots by Jung et al., ${ }^{9}$ where the ground state of only one of the dots was perturbed by a pulsed RF field. This ground-state perturbation introduced an asymmetry in the initial conditions and, hence, the system behaved like coupled harmonic oscillators where the magnetic potential energy of a vortex core was transferred from one dot to the other. Therefore, the transfer of the vortex potential energy observed in our case for the chaotic regime (as opposed to the linear regime) is unique. When the amplitude of the driving signal is reduced, the number of vortex core reversal events decreases and the rotation of both vortex cores eventually become steady without any further reversals (pink region in Fig. 3b,c).
For the simulated responses shown in Fig. 3, the structure was excited at a fixed driving frequency of $530 \mathrm{MHz}$, which is the resonance frequency of the $p_{1} p_{2}=-1$ mode. Therefore, for this driving frequency, the ground state with opposite polarities of the interacting vortex cores is more susceptible to the core reversal. By contrast, for the ground state with the same polarities of the vortex cores (and having a different eigenfrequency), a substantially larger driving amplitude is required to induce the vortex core reversal. This resonance asymmetry is responsible for our ability to select a particular dynamic collective mode (and a corresponding particular configuration of the vortex core polarities). As a result of the initialization process described above, after passing through a quasi-chaotic regime of continuous core reversals followed by a subsequent decrease of the driving amplitude, the system settles into a regime of steady-state vortex gyration with the mode whose eigenfrequency is non-resonant with the driving frequency.

RF power dependence on polarity control. In the experiments we applied a fixed high-amplitude driving signal of $P_{\text {High }}$ to induce the vortex core reversals. At the same time, the simulation results clearly indicate that there is a certain threshold in the driving power, below which the mode selection procedure does not work. To experimentally verify this, we subjected our doubledot samples to different amplitudes of driving fields. The experimentally measured colour-coded frequency spectra of the system subjected to the driving frequencies of $\omega_{\uparrow \downarrow}=265 \mathrm{MHz}$ and $\omega_{\uparrow \uparrow}=305 \mathrm{MHz}$ are summarized in Fig. $4 \mathrm{a}$,b, respectively. To obtain these spectra, we varied the value of $P_{\text {high }}$ during the initialization process at fixed excitation frequencies of 265 and $305 \mathrm{MHz}$. Low-power frequency scans were then performed to identify the collective modes present in the system. The horizontal dotted lines indicate the critical driving amplitudes above which one of the collective modes in the system completely 
a

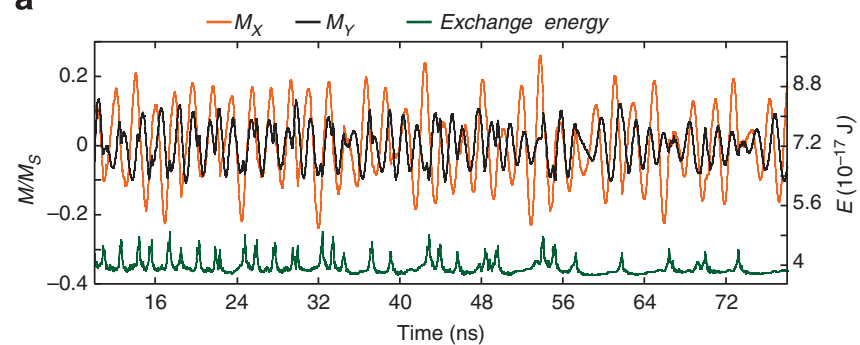

b

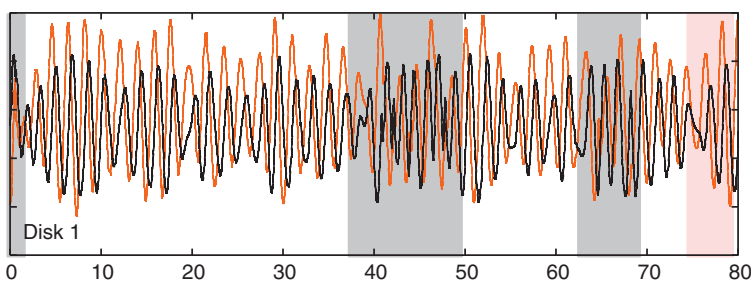

C

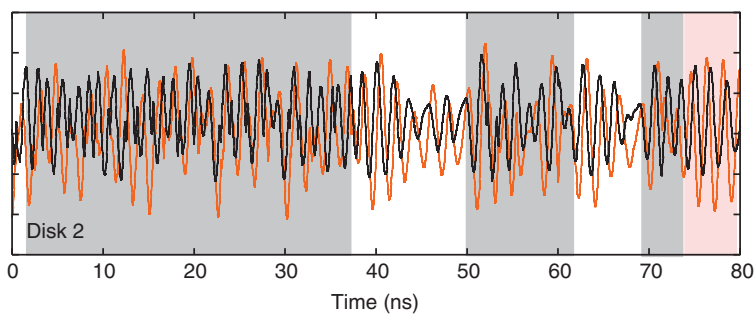

d
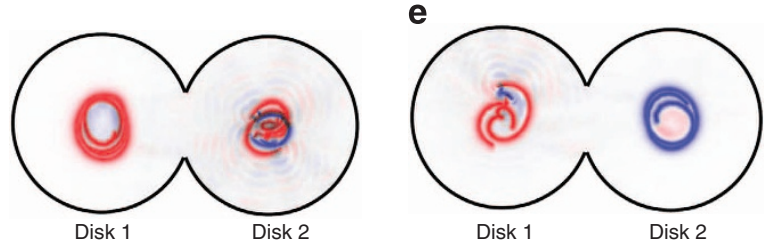

Figure 3 | Transition region between chaos and order. (a) Time-

dependent average magnetization and exchange energy response of the transition region shown in Fig. 2 b. $(\mathbf{b}, \mathbf{c})$ The computed magnetization responses from individual disks. The grey region corresponds to the vortex core reversal in a particular disk. The vortex core trajectories in each disk when the vortex core reversal occurs in only one disk are shown in $\mathbf{d}$ and $\mathbf{e}$. Presence of only one colour in a disk (either red or blue) indicates constant gyration of the vortex core (as shown in disk 1 of $\mathbf{d}$ and disk 2 of $\mathbf{e}$ ). Vortex core reversal is marked by two colours in the disk for the original and reversed polarity of the core (disk 2 in $\mathbf{d}$ and disk 1 in $\mathbf{e}$ ).

disappears. For example, in Fig. 4a, where the driving frequency is $\omega_{\uparrow \downarrow}=265 \mathrm{MHz}$, a driving power of $<-2 \mathrm{dBm}$ is not sufficient to induce vortex core reversals and, consequently, both absorption peaks are present in the spectrum. However, for driving powers $>-2 \mathrm{dBm}$, the excitation amplitude is sufficiently strong to induce the core reversal process and, consequently, only one collective mode (not resonant with the driving) is present. Figure $4 \mathrm{~b}$ (where the driving frequency is $\omega_{\uparrow \uparrow}=305 \mathrm{MHz}$ ) shows the same effect, but with a different collective mode as the resultant ground state. The difference in the critical driving amplitudes (shown by dotted lines) for these two distinct cases is a direct measure of the strength of the dynamical interaction between the collective gyrotropic modes in our double-dot sample.

Thus, two collective gyrotropic modes with substantially different frequencies were observed in the two-vortex model system. Our experiment clearly demonstrated that the final ground state of the system, which was achieved after the initialization process, is only dependent on the driving frequency and not on the initial ground state of the system (that is, the initial combination of the vortex core polarities). The mode that is present at the end of the process is non-resonant with the driving field, and it is consequently completely decoupled from the driving field.

Dependence of resultant polarity combination on external magnetic field. The frequencies of collective modes are strongly dependent on the external magnetic field. Consequently, varying the bias field can be used to tune the eigenfrequencies of the system. Thus, a particular collective mode can be coupled or decoupled to RF input signal of given (fixed) frequency by varying the strength of the external field when applied along the $y$ axis of the system. This process is illustrated in Supplementary Fig. S1, where a particular collective mode can be selected by choosing a proper field sweep range. On the basis of the universality of the mode selection phenomena, a phase diagram has been constructed with respect to the excitation frequency and the external magnetic field, as shown in Fig. 4c. This experimental phase diagram for the connected double-dot structure specifically provides the desired parameters for defining the ground state with either of the two polarity combinations. An external magnetic field can be swept horizontally on the phase diagram at a constant excitation frequency and, depending on the direction of the sweep and on the stop field, a unified state can be set in the entire array. Similarly, the frequency can be swept vertically in the presence of a constant magnetic field to obtain a specific ground state.

\section{Discussion}

We developed a new and effective method to control the ground state determined by the relative vortex core polarities and the dynamic excitation mode in a model system of coupled vortexstate magnetic dots. The method involves driving the system by a strong periodic driving field into a quasi-chaotic regime of continuous vortex core reversals. This process is followed by a gradual reduction of the driving field and relaxing of the system to a ground state, which supports the dynamical mode that is non-resonant with the driving frequency and is therefore nonresponsive with the driving field. A similar approach can be used to dynamically select a particular ground state in a vortex-state magnonic crystal $^{12,13}$ and in other physical systems that have multiple stable ground states, such as arrays of coupled nanooscillators $^{26,27}$ or spin-ice-type lattices ${ }^{28}$. Furthermore, we believe that the proposed approach to control the remanent state by exploring driven (non-linear) dynamics can be applied to nonmagnetic systems that are characterized by ground states.

\section{Methods}

Sample fabrication. The samples were fabricated using electron-beam lithography and lift-off processes. The double-dot structure has dimensions of $2 \times 1 \mu \mathrm{m}^{2}$ with vertical contact length of $740 \mathrm{~nm}$ and thickness $48 \mathrm{~nm}$. The separation between each pair is $1 \mu \mathrm{m}$ and, therefore, dipole-dipole interactions between dot pairs can be neglected. To measure the resonance frequencies of the gyrotropic modes, the structures were fabricated on top of a shorted $\mathrm{Cr}(10 \mathrm{~nm}) / \mathrm{Au}(300 \mathrm{~nm}) \mathrm{CPW}$ with a $3-\mu \mathrm{m}$-wide signal line.

Micromagnetic simulations. Micromagnetic simulation $\mathrm{s}^{29}$ were performed using the standard $\mathrm{Ni}_{80} \mathrm{Fe}_{20}$ parameters; an exchange stiffness constant of $A=13 \mathrm{pJ} \mathrm{m}-1$, saturation magnetization $\mathrm{Ms}=8 \times 10^{5} \mathrm{~A} \mathrm{~m}^{-1}$, gyromagnetic ratio $\gamma=18.5 \mathrm{GHz}$ per $\mathrm{kOe}$, a dimensionless Gilbert constant $\alpha=0.01$, cell size of $5 \times 5 \times 5 \mathrm{~nm}^{3}$ and magnetocrystalline anisotropy was neglected. To avoid large computational times, the structural dimensions were reduced by half to $1 \times 0.5 \mu \mathrm{m}^{2}$ rather than $2 \times 1 \mu \mathrm{m}^{2}$. The resonance frequencies of the system are obtained by sweeping the driving frequency from 300 to $900 \mathrm{MHz}$ with a constant RF field amplitude of 1 Oe. The representative time evolution of the two magnetization components for both the polarity combinations $\left(p_{1} p_{2}= \pm 1\right)$ are presented in Supplementary Fig. S2. The 
a

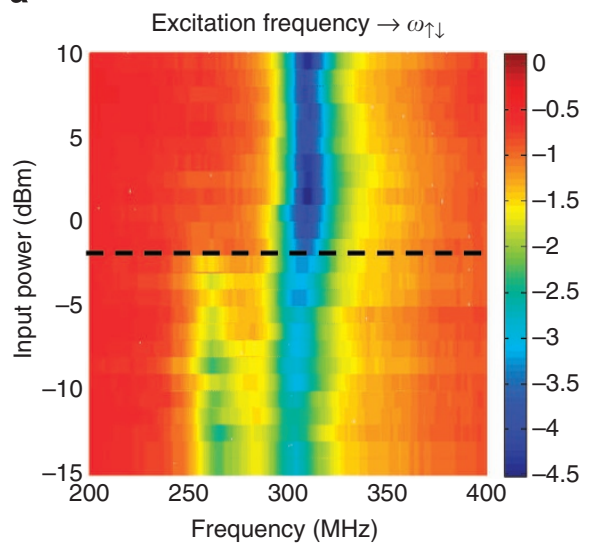

b

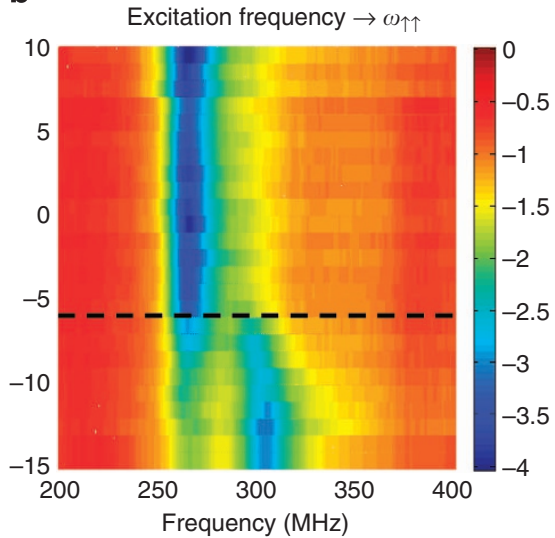

C

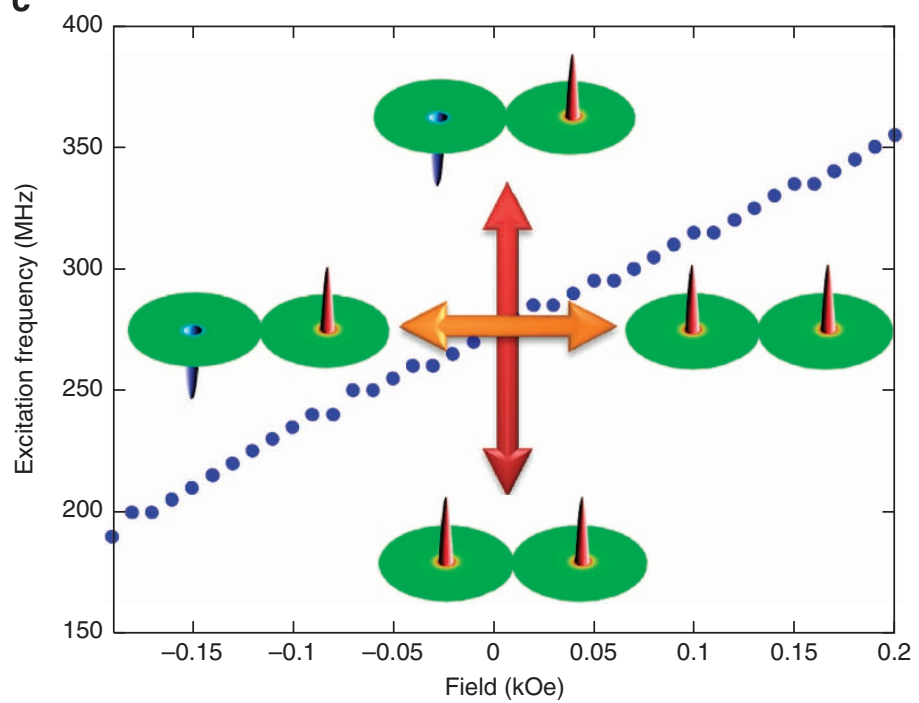

Figure 4 | Drive amplitude, frequency and magnetic field dependence on mode selection. Frequency spectrum obtained at $-15 \mathrm{dBm}\left(P_{\mathrm{Low}}\right)$ and $230 \mathrm{MHz}$ for various high RF-amplitudes at constant excitation frequencies of (a) $264 \mathrm{MHz}$ and (b) $304 \mathrm{MHz}$. The dashed lines in $\mathbf{a}$ and $\mathbf{b}$ correspond to the critical threshold magnitude of input power, which is required to completely suppress the resonant mode. (c) Universal phase diagram constructed for the excitation frequency versus external magnetic field. The circular dots mark the transition region where selecting a specific field direction (left $\rightarrow$ right or right $\rightarrow$ left) at a fixed excitation frequency will yield a desired polarity combination in the disks. Conversely, vertical arrows that the same polarity combination can also be chosen by keeping the magnitude of external magnetic field constant and sweeping the excitation frequency.

calculated eigenfrequencies of the two modes are $530\left(p_{1} p_{2}=-1\right)$ and $580 \mathrm{MHz}$ $\left(p_{1} p_{2}=+1\right)$. Qualitative analysis of the collective vortex modes was also performed. The details of this analysis are provided in Supplementary Methods.

\section{References}

1. Nardin, G. et al. Hydrodynamic nucleation of quantized vortex pairs in a polariton quantum fluid. Nat. Phys. 7, 635-641 (2011).

2. Khaykovich, L. et al. Formation of a matter-wave bright soliton. Science 296, 1290-1293 (2002).

3. Abrikosov, A. A. On the magnetic properties of supercondictors of the second group. J. Exptl. Theoret. Phys. 32, 1442-1452 (1957).

4. Novosad, V. et al. Nucleation and annihilation of magnetic vortices in submicron permalloy dots. IEEE Trans. Magn. 37, 2088-2090 (2001).

5. Choe, S.-B. et al. Vortex core-driven magnetization dynamics. Science 304, 420-422 (2004).

6. Novosad, V. et al. Magnetic vortex resonance in patterned ferromagnetic dots. Phys. Rev. B 72, 024455 (2005).

7. Guslienko, K. et al. Eigenfrequencies of vortex state excitations in magnetic submicron-size disks. J. Appl. Phys. 91, 8037-8039 (2002).

8. Sugimoto, S. et al. Dynamics of coupled vortices in a pair of ferromagnetic disks. Phys. Rev. Lett. 106, 197203 (2011)

9. Jung, H. et al. Tunable negligible-loss energy transfer between dipolar-coupled magnetic disks by stimulated vortex gyration. Sci. Reports 1, 59 (2011).
10. Vogel, A., Drews, A., Kamionka, T., Bolte, M. \& Meier, G. Influence of dipolar interaction on vortex dynamics in arrays of ferromagnetic disks. Phys. Rev. Lett. 105, 037201 (2010).

11. Awad, A. A. et al. Spin excitation frequencies in magnetostatically coupled arrays of vortex state circular permalloy dots. Appl. Phys. Lett. 97, 132501 (2010).

12. Huber, R. \& Grundler, D. Ferromagnetic nanodisks for magnonic crystals and waveguides. Proc. SPIE 8100, 81000D (2011).

13. Shibata, J. \& Otani, Y. Magnetic vortex dynamics in a two-dimensional square lattice of ferromagnetic nanodisks. Phys. Rev. B 70, 012404 (2004).

14. Verba, R., Melkov, G., Tiberkevich, V. \& Slavin, A. Collective spin-wave excitations in a two-dimensional array of coupled magnetic nanodots. Phys. Rev. B 85, 014427 (2012).

15. Verba, R., Melkov, G., Tiberkevich, V. \& Slavin, A. Fast switching of a ground state of a reconfigurable array of magnetic nano-dots. Appl. Phys. Lett. 100, 192412 (2012).

16. Yakata, S. et al. Chirality control of magnetic vortex in a square Py dot using current-induced Oersted field. Appl. Phys. Lett. 99, 242507 (2011).

17. Jaafar, M. et al. Control of the chirality and polarity of magnetic vortices in triangular nanodots. Phys. Rev. B 81, 05439 (2010).

18. Waeyenberge, B. et al. Magnetic vortex core reversal by excitation with short bursts of an alternating field. Nature 444, 461-464 (2006).

19. Pigeau, B. et al. Optimal control of vortex-core polarity by resonant microwave pulses. Nat. Phys. 7, 26-31 (2011).

20. Kikuchi, N. et al. Vertical bistable switching of spin vortex in a circular magnetic dot. J. Appl. Phys. 90, 6548-6549 (2001). 
21. Curcic, M. et al. Magnetic vortex core reversal by rotating magnetic fields generated on micrometer length scales. Phys. Status Solidi B 248, 2317-2322 (2011).

22. Liu, Y., Gliga, S., Hertel, R. \& Schneider, C. M. Current-induced magnetic vortex core switching in a permalloy nanodisk. Appl. Phys. Lett. 91, 112501 (2007).

23. Yamada, K. et al. Electrical switching of the vortex core in a magnetic disk. Nat. Mater. 6, 269-273 (2007).

24. Buchanan, K. S. et al. Soliton-pair dynamics in patterned ferromagnetic ellipses. Nat. Phys. 1, 172-176 (2005).

25. Gliga, S., Liu, Y. \& Hertel, R. Energy thresholds in the magnetic vortex core reversal. J. Phys. 303, 012005 (2011)

26. Dussaux, A. et al. Large microwave generation from current-driven magnetic vortex oscillators in magnetic tunnel junctions. Nat. Commun. 1, 8 (2010)

27. Pribiag, V. S. et al. Magnetic vortex oscillator driven by d.c. spin-polarized current. Nat. Phys. 3, 498-503 (2007).

28. Wang, R. F. et al. Artificial spin ice in a geometrically frustrated lattice of nanoscale ferromagnetic islands. Nature 439, 303-306 (2006).

29. Donahue, M. J. \& Porter, D. G. OOMMF User's guide, Version 1.0, Interagency Report NIST IR 6376 (Gaithersburg, MD, 1999).

\section{Acknowledgements}

Work at Argonne and use of the Center for Nanoscale Materials was supported by the US Department of Energy, Office of Science, Office of Basic Energy Sciences, under Contract
No. DE-AC02-06CH11357. This work was in part supported by grant DMR-1015175 from the US National Science Foundation, by a Contract from the US Army TARDEC and RDECOM, and by a grant from DARPA.

\section{Author contributions}

S.J. and V.N. conceived the idea, fabricated samples, performed experiments and micromagnetic modelling. F.Y.F. and J.E.P. setup the measurement apparatus. F.Y.F. designed and modelled microwave properties of the waveguide structures. S.J. and J.E.P. deposited magnetic thin films. A.S. and V.T. developed the analytical theory. S.J., V.N., F.Y.F, J.E.P., A.S., V.T. and S.D.B. discussed the results and wrote the text of the manuscript.

\section{Additional information}

Supplementary Information accompanies this paper at http://www.nature.com/ naturecommunications

Competing financial interests: The authors declare no competing financial interests.

Reprints and permission information is available online at http://npg.nature.com/ reprintsandpermissions/

How to cite this article: Jain, S. et al. From chaos to selective ordering of vortex cores in interacting mesomagnets. Nat. Commun. 3:1330 doi: 10.1038/ncomms2331 (2012). 